\title{
CT-guided Ultrathin Bronchoscopy: Bioptic Approach and Factors in Predicting Diagnosis
}

\author{
Yoshihiko Matsuno ${ }^{1}$, Fumihiro Asano ${ }^{1}$, Joe Shindoh ${ }^{2}$, Takashi Abe $^{2}$, Akira Shiraki ${ }^{2}$, \\ Morihide Ando $^{2}$, Toshitaka Suzuki ${ }^{1}$, Akira Seko ${ }^{1}$ and Hisataka Moriwaki ${ }^{3}$
}

\begin{abstract}
Background The diagnostic sensitivity of current bronchoscopy for peripheral lung cancer is inadequate because the bronchoscope insertion range is limited and confirmation of the position of the biopsy apparatus at the lesion under X-ray fluoroscopy is inaccurate. The combination of ultrathin bronchoscopy and computed tomography (CT) is effective for solving these problems.

Objective This study was a retrospective study analyzing prospectively collected data to identify factors contributing to the diagnosis and the appropriate biopsy method in CT-guided ultrathin bronchoscopy for peripheral lung cancer.

Methods The subjects comprised 86 patients (88 lesions) who underwent CT-guided ultrathin bronchoscopy and were finally diagnosed with peripheral lung cancer. We evaluated the diagnostic yield according to specific factors and also according to the sample collection method.

Results Sixty-nine lesions were diagnosed as lung cancer, and the diagnostic yield was $78.4 \%(80.3 \%$ in lesions $\leq 2 \mathrm{~cm}$ in diameter). Multivariate analysis showed that the factors contributing to the diagnosis were the observation range by ultrathin bronchoscopy and the presence/absence of the involved bronchus or pulmonary artery. Pathological evaluation facilitated histological diagnoses in $53(65.4 \%)$ of 81 lesions. In 16 lesions, only the cytological diagnosis was positive.

Conclusion CT-guided ultrathin bronchoscopy may be particularly useful for lesions for which the involved bronchus or pulmonary artery can be confirmed, and observation of bronchi of the 6th generation or more is possible. Since the specimen preparation rate is low, the combination of histopathological diagnosis with cytological diagnosis particularly that of the discharge attached to the forceps, is optimal.
\end{abstract}

Key words: cytology, lung cancer, transbronchial biopsy, ultrathin bronchoscope, virtual bronchoscopy

(Intern Med 50: 2143-2148, 2011)

(DOI: 10.2169/internalmedicine.50.5666)

\section{Introduction}

With the widespread use of computed tomography (CT), cases of peripheral lung cancer have been more frequently detected. Bronchoscopy is a routine diagnostic method for these lesions, with few related complications. The ACCP guidelines have shown that the diagnostic sensitivity of bronchoscopy for peripheral lung cancer is 0.78 , but only 0.34 for lesions with a diameter $\leq 2 \mathrm{~cm}$, which is inadequate (1).
In general, bronchoscopy for peripheral lung cancer is performed using a bronchoscope with an outer diameter of about $5 \mathrm{~mm}$ under X-ray fluoroscopy. The problems with this method are as follows: 1) confirmation of the position of the biopsy apparatus at the lesion is inaccurate under $\mathrm{X}$ ray fluoroscopy; and 2) since the insertion range of the bronchoscope itself and the resulting bronchoscopic observation range are limited, guidance of the biopsy apparatus to the lesion is difficult. To overcome the first problem, the combination of bronchoscopy with CT fluoroscopy (2), endobronchial ultrasonography (EBUS) (3), or electromagnetic

${ }^{1}$ Department of Internal Medicine, National Health Insurance Sekigahara Hospital, Japan, ${ }^{2}$ Department of Respiratory Medicine, Ogaki Municipal Hospital, Japan and ${ }^{3}$ First Department of Internal Medicine, Gifu University Graduate School of Medicine, Japan

Received for publication April 18, 2011; Accepted for publication June 27, 2011

Correspondence to Dr. Fumihiro Asano, asano-fm@ceres.ocn.ne.jp 
navigation (EMN) (4) has been reported. To ameliorate the second problem, the bronchoscope diameter has been reduced. Ultrathin bronchoscopes were initially used for observation mainly in children, but have recently begun to be used to examine adults because they enable biopsy to be performed (5-8). Ultrathin bronchoscopes can be advanced to more peripheral bronchi than conventional bronchoscopes (9). Because ultrathin bronchoscopes can be advanced close to peripheral lesions, the subsequent guidance of the forceps is easy. Ultrathin bronchoscopes are useful for the diagnosis of peripheral pulmonary lesions, particularly those in $\mathrm{S} 1$ and $\mathrm{S} 1+2$, into which insertion of conventional bronchoscopes is limited (10). To make use of this advantage, we have proposed a method in which a bronchoscope is navigated using virtual bronchoscopic images en route to the lesion (virtual bronchoscopic navigation: VBN) (11). For a method that combines measures addressing the two abovementioned problems stated, Kobayashi et al reported CTguided ultrathin bronchoscopy (12). Using the combination of ultrathin bronchoscopy and CT, the position of the biopsy apparatus at the lesion can be more accurately confirmed. CT-guided bronchoscopy does, however, expose the patient to X-rays. In addition, while the bronchoscopy is being performed, the CT equipment cannot be used for other procedures, so potential patients should be chosen with this in mind. Also, since the biopsy apparatus that can be used with ultrathin bronchoscopes is small, adequate samples are sometimes difficult to obtain (9). There have been studies on the sample collection method in bronchoscopy (1), but none on the use of ultrathin bronchoscopes. To identify factors contributing to the diagnosis and the appropriate biopsy method in CT-guided ultrathin bronchoscopy, we evaluated the diagnostic yield according to factors and pathological findings in cases that were ultimately diagnosed as lung cancer.

\section{Materials and Methods}

\section{Subjects}

Between July 1999 and March 2005, CT-guided ultrathin bronchoscopy was prospectively performed in 166 lesions in 162 patients (105 males and 57 females; median age, 70.3 years) at the National Health Insurance Sekigahara Hospital and Ogaki Municipal Hospital. This bronchoscopy was performed for peripheral pulmonary lesions fulfilling one of the following criteria: 1) lesions that could not be observed by X-ray fluoroscopy; 2) those with a diameter $\leq 2 \mathrm{~cm}$; and 3) those that could not be diagnosed by conventional bronchoscopy under X-ray fluoroscopy. Peripheral pulmonary lesions were defined as lesions that were surrounded by pulmonary parenchyma and endoscopically invisible lesions. Lesions with a CT-confirmed pure ground-glass opacity pattern $(\leq 10 \mathrm{~mm})$ were excluded because of the difficulty in diagnosing them by biopsy, which allows the collection of only part of the lesion. Informed consent was obtained from all patients. Lesions not diagnosed by CT-guided ultrathin bronchoscopy were subsequently diagnosed by transthoracic biopsy, surgery, or follow-up of the clinical course for 2 years or more. Cases ultimately diagnosed as primary lung cancer were analyzed. The institutional review board for human research approved the study protocol.

\section{Methods}

\section{CT}

CT examination was performed using a multidetector CT scanner with $0.5-1-\mathrm{mm}$ collimation. All lung fields were scanned during a single breath-holding period. Axial thinsection CT (TSCT) images were obtained by reconstruction at $0.5-\mathrm{mm}$ intervals. Axial TSCT images were evaluated by two experienced pulmonologists to assess the relationship between the lesions and bronchial or arterial trees. In some cases, for bronchoscopic navigation, based on the helical CT data, virtual bronchoscopy (VB) was performed using software (Navigator, Advantage Windows 2.0: GE Medical Systems, Tokyo, Japan), and VB images to the lesion were produced.

\section{CT-guided ultrathin bronchoscopy}

Bronchoscopy was performed using an ultrathin bronchoscope (BF-type XP40 or XP260F, Olympus Co., Ltd., Tokyo, Japan: external diameter, $2.8 \mathrm{~mm}$; channel diameter, $1.2 \mathrm{~mm}$ ) in a room equipped with $\mathrm{CT}$ and X-ray fluoroscopy apparatuses. Each patient was premedicated with 25 $\mathrm{mg}$ hydroxyzine and $0.5 \mathrm{mg}$ atropine sulfate. Local anesthesia of the upper respiratory tract was performed using $2 \%$ lidocaine. Referring to TSCT images or VB images, the ultrathin bronchoscope was inserted into the target bronchus as far as possible under direct vision. A pair of forceps (FD44D-1, FB-56D-1, Olympus Co., Ltd., Tokyo, Japan) was then guided to the lesion under X-ray fluoroscopy, and, if necessary, CT fluoroscopy (tube voltage, $120 \mathrm{kV}$; tube current, $30 \mathrm{~mA}$; collimation thickness, $5 \mathrm{~mm}$ ). TSCT was performed under the same conditions as those for TSCT performed before bronchoscopy, except for the tube current (50 $\mathrm{mA}$ ), to determine whether the forceps had reached the lesion. At this time, the position of the forceps was also confirmed by X-ray fluoroscopy and recorded, and biopsy was subsequently repeated under X-ray fluoroscopy. When the ultrathin bronchoscope or biopsy forceps were displaced, CT fluoroscopy was used for re-confirmation if necessary. Biopsy was then repeated until adequate histological specimens were collected. Whenever possible, we also performed cytological diagnosis of the discharge attached to the biopsy forceps, substances that were brushed off using a brush (BC-201C-1006, BC203D-2006 Olympus Co., Ltd., Tokyo, Japan), and bronchial lavage fluid. Specifically, a histological specimen was collected from the lesion using a biopsy forceps. The collected specimen was immediately fixed in formalin. After removal of the specimen from the forceps, the discharge attached to the forceps was smeared onto a 


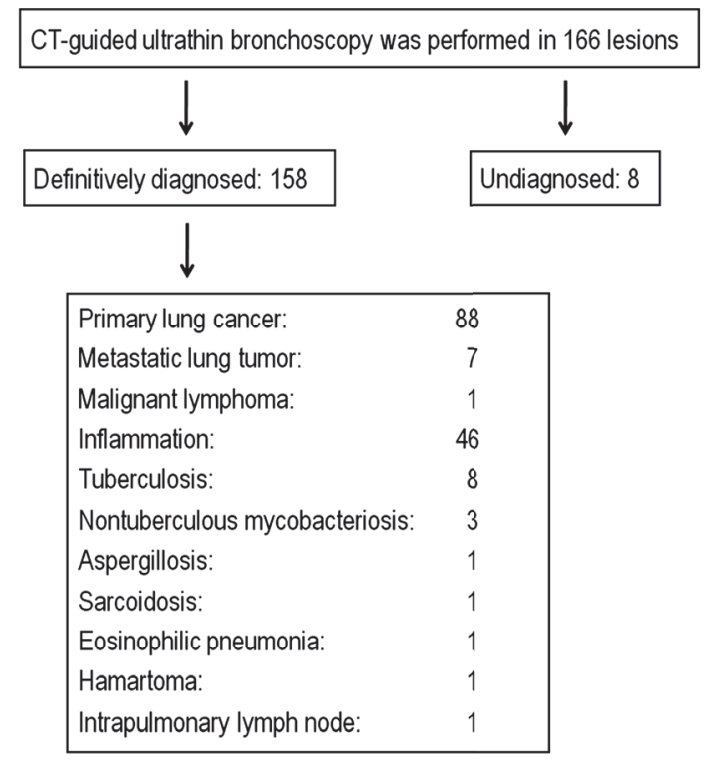

Figure 1. Subjects of CT-guided ultrathin bronchoscopy and definitive diagnosis.

slide for cytological diagnosis and immediately fixed in alcohol. Subsequently, the lesion was brushed, and finally the area around the lesion was washed with $20 \mathrm{~mL}$ of saline. The cytological and histological diagnoses were evaluated independently. The presence of bacteria was assessed in some portions of the lavage.

\section{Statistical analysis}

All data were processed using standard statistical methods. The results are presented as the median and range. Data were analyzed using Pearson's X2 test. Univariate logistic analysis of each factor was performed, and p-value, odds ratio, and $95 \%$ confidence interval were obtained. Multivariate logistic analysis of factors showing a $p$-value $\leq 0.2$ by univariate logistic analysis was performed, $\mathrm{p}$-value, odds ratio, and the $95 \%$ confidence interval were similarly obtained, and factors associated with the diagnosis were selected. To exclude the influences of correlations among factors, the stepwise method was used. Statistical software (SAS version 9.1.3 for Windows; SAS Institute, Cary, NC) was employed for all analyses. Statistical significance was established at the level of $\mathrm{p}<0.05$, and all analyses were two-sided.

\section{Results}

CT-guided ultrathin bronchoscopy was performed in 162 patients (166 lesions). As a complication, pneumothorax was observed in 1 case $(0.6 \%)$, but improved after course observation. Eight patients ( 8 lesions) dropped out. Figure 1 shows the final diagnoses in the remaining 154 patients (158 lesions). The final diagnosis was made based on the findings of surgery, percutaneous biopsy, and follow-up observation. Cases showing no specific lesions and no changes, a decrease, or disappearance of the lesion after follow-up observation for 2 years or more were classified as inflammation.
There were 8 malignant lesions other than primary lung cancer ( 7 metastatic lung tumors and 1 malignant lymphoma), of which 6 (75\%) were diagnosed using CT-guided ultrathin bronchoscopy. There were 62 benign lesions, of which 38 $(61.3 \%)$ were diagnosed using CT-guided ultrathin bronchoscopy. Analysis was performed in 86 patients ( 88 lesions) in whom the final diagnosis was primary lung cancer.

The patients analyzed consisted of 57 males $(66.3 \%)$ and 29 females $(33.7 \%)$, with a median age of 71.5 years (49-86 years). The median lesion size was $16.25 \mathrm{~mm}(7-40 \mathrm{~mm})$. The lesion was located in the right superior lobe in 27 lesions $(30.7 \%)$, right middle lobe in $7(8.0 \%)$, right inferior lobe in $18(20.5 \%)$, left superior lobe in 17 (19.3\%), left inferior lobe in $19(21.6 \%)$, and could not be confirmed by X ray radiography in $41(46.6 \%)$. Thin-section CT showed a GGO pattern in 12 lesions (13.6\%) and could identify the involved bronchus or pulmonary artery in $62(70.5 \%)$. The histological type was adenocarcinoma in 68 lesions (77.3\%), squamous cell carcinoma in $11(12.5 \%)$, small cell carcinoma in $3(3.4 \%)$, large cell carcinoma in $1(1.1 \%)$, and unknown in $5(5.7 \%)$. VBN was used for guidance of the bronchoscope in 52 lesions $(59.1 \%)$. Using the ultrathin bronchoscope, observation up to a median of the 6thgeneration bronchi (range, 3rd-9th bronchi) was possible, and the bronchus for insertion of the forceps could be selected under direct vision. Observation up to bronchi of the 6 th generation or more was possible in 55 lesions $(62.5 \%)$. In compliance with the nomenclature of the Japan Society of Bronchology, segmental bronchi were regarded as 2ndgeneration bronchi and subsegmental bronchi, as 3rdgeneration bronchi.

CT confirmed the position of the biopsy apparatus at the lesion in $77(87.5 \%)$ of the 88 lesions, and 69 lesions (78.4\%) were diagnosed as lung cancer by CT-guided bronchoscopy. The tumor itself could not be observed in the visible range in any patient. In 11 of the 19 lesions that could not be diagnosed, the position of the biopsy apparatus at the lesion could not be confirmed by CT. The diagnostic yield according to lesion size was $53.3 \%(8 / 15)$ for diameters $\leq$ $10 \mathrm{~mm}, 78.3 \%(18 / 23)$ for $>10-15 \mathrm{~mm}, 96.4 \%$ (27/28) for $>$ $15-20 \mathrm{~mm}$, and $72.7 \%(16 / 22)$ for $>20 \mathrm{~mm}$. The diagnostic yields according to the segment and bronchoscopic observation range are shown in Table 1. According to the histological type, the diagnostic yield was $77.9 \%$ (53/68) for adenocarcinoma, $90.9 \%(10 / 11)$ for squamous cell carcinoma, $66.7 \%(2 / 3)$ for small cell carcinoma, $0 \%(0 / 1)$ for large cell carcinoma, and $80 \%(4 / 5)$ for the unknown type.

The diagnostic yields according to factors are shown in Table 2. Univariate analysis revealed significant differences in the diagnostic yield between the presence and absence of a GGO CT pattern, the presence and absence of the involved bronchus or pulmonary artery on CT images, and the observation range by ultrathin bronchoscopy (bronchi $\geq 6$ th generation and those $<6$ th generation). Multivariate analysis was performed using items showing a $p$-value $\leq 0.2$. Factors contributing to the diagnosis were the observation range by 
Table 1. Diagnostic Yield and Bronchial Generation of Observation Range

\begin{tabular}{|c|c|c|c|c|c|}
\hline Segment & $\begin{array}{l}\text { No. of total } \\
\text { lesions }\end{array}$ & $\begin{array}{c}\text { No. of } \\
\text { diagnosed } \\
\text { lesions }\end{array}$ & Yield (\%) & \multicolumn{2}{|c|}{$\begin{array}{c}\text { Bronchial generation of } \\
\text { observation range }\end{array}$} \\
\hline RUL & 27 & 21 & $77.8 \%$ & 6 & $(4-9)$ \\
\hline B1 & 10 & 7 & $70.0 \%$ & 5 & $(4-8)$ \\
\hline B2 & 8 & 8 & $100.0 \%$ & 6.5 & $(5-8)$ \\
\hline B3 & 9 & 6 & $66.7 \%$ & 7 & $(5-9)$ \\
\hline RML & 7 & 7 & $100.0 \%$ & 7 & $(4-9)$ \\
\hline B4 & 5 & 5 & $100.0 \%$ & 8 & $(6-9)$ \\
\hline B5 & 2 & 2 & $100.0 \%$ & 4 & (4) \\
\hline RLL & 18 & 15 & $83.3 \%$ & 6 & $(5-8)$ \\
\hline B6 & 9 & 9 & $100.0 \%$ & 7 & $(5-8)$ \\
\hline B7 & 1 & 0 & $0.0 \%$ & 5 & (5) \\
\hline B8 & 4 & 3 & $75.0 \%$ & 6.5 & $(5-8)$ \\
\hline B9 & 3 & 2 & $66.7 \%$ & 5 & $(5-7)$ \\
\hline B10 & 1 & 1 & $100.0 \%$ & 6 & (6) \\
\hline LUL & 17 & 13 & $76.5 \%$ & 6 & $(4-8)$ \\
\hline $\mathrm{B} 1+2$ & 6 & 5 & $83.3 \%$ & 6.5 & $(4-8)$ \\
\hline B3 & 6 & 4 & $66.7 \%$ & 6 & $(4-8)$ \\
\hline B4 & 2 & 1 & $50.0 \%$ & 6.5 & $(6-7)$ \\
\hline B5 & 3 & 3 & $100.0 \%$ & 5 & $(4-6)$ \\
\hline LLL & 19 & 13 & $68.4 \%$ & 5 & $(3-8)$ \\
\hline B6 & 7 & 4 & $57.1 \%$ & 5 & $(4-6)$ \\
\hline B8 & 3 & 2 & $66.7 \%$ & 6 & $(4-6)$ \\
\hline B9 & 4 & 2 & $50.0 \%$ & 6.5 & $(6-7)$ \\
\hline B10 & 5 & 5 & $100.0 \%$ & 4 & $(3-8)$ \\
\hline Total & 88 & 69 & $78.4 \%$ & 6 & $(3-9)$ \\
\hline
\end{tabular}

Data are presented as the median, and the range in parentheses.

$\mathrm{RUL}=$ right upper lobe; $\mathrm{RML}$ = right middle lobe; $\mathrm{RLL}=$ right lower lobe; $\mathrm{LUL}=$ left upper lobe; LLL = left lower lobe.

ultrathin bronchoscopy ( $\mathrm{p}=0.039$; odds ratio, $3.46 ; 95 \%$ Wald confidence limits, 1.07-11.22) and the presence/absence of the involved bronchus or pulmonary artery $(\mathrm{p}<$ 0.001: odds ratio, 8.65; 95\% Wald confidence limits, 2.6628.10).

Concerning pathological evaluation, a total of 415 biopsy procedures (median, 5 procedures per lesion; range, 1-11 per lesion) were performed in 81 of the 88 lesions, and, finally, 287 biopsy histological specimens were obtained (median, 3 specimens per lesion; range, 1-9 per lesion); the specimen preparation rate was $69.2 \%$ (287/415). Fifty-three of these 81 lesions $(65.4 \%)$ were histologically diagnosed as lung cancer.

Cytological diagnosis was also performed in 82 lesions and was positive in $61(74.4 \%)$. The positivity rate according to the sample collection method was $68.0 \%$ (51/75) for the cytological diagnosis of discharge attached to the forceps, $45.9 \%$ (28/61) for brush cytological diagnosis and $29.0 \%(20 / 69)$ for lavage cytological diagnosis. Among the lesions evaluated by these cytological diagnostic methods, 16 could not be histologically diagnosed and were only positive for the cytological diagnosis. Of the 16 lesions, 8 were only positive for cytological diagnosis of the discharge attached to the forceps, and 2 were positive only for brush cytological diagnosis; there were no lesions positive only for lavage cytological diagnosis.

\section{Discussion}

This study was the first to show the high diagnostic yield (78.4\%) of CT-guided ultrathin bronchoscopy for primary lung cancer, and clarify that the CT-bronchus and/or artery sign and the observation range of ultrathin bronchoscopy are factors contributing to diagnosis using multivariate analysis. In addition, we were the first to evaluate the specimen sampling method in ultrathin bronchoscopy, and show the advantage of the combined use of cytology, particularly cytological diagnosis of the discharge attached to biopsy forceps.

The diagnostic yield by transbronchial biopsy is dependent upon the characteristics of the lesions $(1,13,14)$. In this study, lesions that are generally difficult to diagnose, such as those that are small, cannot be visualized by X-ray fluoroscopy, or cannot be diagnosed by previous conventional bronchoscopy, were evaluated. Considering this, the diagnostic rate of $78.4 \%$, and particularly that of $80.3 \%$ for lesions $\leq 20 \mathrm{~mm}$, appeared to be high. Yamamoto et al (15) performed ultrathin bronchoscopy under X-ray fluoroscopy in 32 lesions that could not be diagnosed by rapid cytological diagnosis using a bronchoscope with a conventional diameter; a diagnosis could be made in 13 (40.6\%), of which 
Table 2. Logistic Analysis of Factors Regarding Diagnostic Yield of CT-guided Ultrathin Bronchoscopy

\begin{tabular}{|c|c|c|c|c|c|c|c|c|c|c|}
\hline & & & No. of & Yjeld & & Univariate & & & Multivariate & \\
\hline & riables & $\begin{array}{c}\text { total } \\
\text { lesions }\end{array}$ & $\begin{array}{l}\text { sed } \\
\text { lesions }\end{array}$ & $(\%)$ & $\begin{array}{l}\text { Odds } \\
\text { ratio }\end{array}$ & $(95 \% \mathrm{Cl})$ & $p$ value & $\begin{array}{l}\text { Odds } \\
\text { ratio }\end{array}$ & $(95 \% \mathrm{Cl})$ & $p$ value \\
\hline Age & $\geq 70$ & 49 & 40 & 81.6 & 1.43 & $0.50-4.05$ & 0.503 & & & \\
\hline Age & $<70$ & 37 & 28 & 75.7 & & & & & & \\
\hline & Male & 57 & 44 & 77.2 & 0.71 & $0.22-2.22$ & 0.550 & & & \\
\hline Sex & Female & 29 & 24 & 82.8 & & & & & & \\
\hline Lesion & $<15$ & 36 & 25 & 69.4 & 0.41 & $0.15-1.16$ & 0.094 & & & \\
\hline diameter & $\geq 15$ & 52 & 44 & 84.6 & & & & & & \\
\hline I cration & $\mathrm{RIL}+\mathrm{RML}$ & 25 & 22 & 88.0 & 2.50 & $0.66-9.47$ & 0.179 & & & \\
\hline Location & Other lobes & 63 & 47 & 74.6 & & & & & & \\
\hline Visibility on & Visible & 47 & 40 & 85.1 & 2.37 & $0.83-6.74$ & 0.107 & & & \\
\hline fluoroscopy & Non-visible & 41 & 29 & 70.7 & & & & & & \\
\hline & GGO & 12 & 6 & 50.0 & 0.21 & $0.06-0.74$ & 0.016 & & & \\
\hline Cl pattern & Non-GGO & 76 & 63 & 82.9 & & & & & & \\
\hline CT-bronchus & Positive & 62 & 56 & 90.3 & 9.33 & $2.98-29.18$ & $<0.001$ & 8.65 & $2.66-28.10$ & $<0.001$ \\
\hline $\begin{array}{l}\text { and/or artery } \\
\text { sign }\end{array}$ & Negative & 26 & 13 & 50.0 & & & & & & \\
\hline Histological & Adenocarcinoma & 68 & 53 & 77.9 & 0.88 & $0.26-3.04$ & 0.844 & & & \\
\hline type & Other type & 20 & 16 & 80.0 & & & & & & \\
\hline Virtual & VB & 52 & 42 & 80.8 & 1.40 & $0.50-3.89$ & 0.519 & & & \\
\hline bronchoscopy & Non-VB & 36 & 27 & 75.0 & & & & & & \\
\hline $\begin{array}{l}\text { Observation } \\
\text { range of }\end{array}$ & $\begin{array}{l}\geq 6 \text { th-generation } \\
\text { bronchus }\end{array}$ & 55 & 48 & 87.3 & 3.92 & $1.35-11.37$ & 0.012 & 3.46 & $1.07-11.22$ & 0.039 \\
\hline $\begin{array}{l}\text { ultrathin } \\
\text { bronchoscope }\end{array}$ & $\begin{array}{l}<6 \text { th-generation } \\
\text { bronchus }\end{array}$ & 33 & 21 & 63.6 & & & & & & \\
\hline
\end{tabular}

9 were located in the superior lobe. In the present patients, the diagnostic yield in lesions that could not be diagnosed by conventional bronchoscopy was $80 \%(8 / 10)$, even higher than in the study of Yamamoto et al. Considering that there was only 1 lesion in the superior lobe, the high diagnostic yield may be due to the use of CT. Tsushima et al (16) reported that the diagnostic yield of malignant disease was $57.1 \%$ by CT-guided bronchoscopy using a bronchoscope with a conventional diameter. Heyer et al (17) reported a diagnostic yield of $72.7 \%$ by CT-guided conventional-diameter bronchoscopy for lesions $2 \mathrm{~cm}$ or more away from the pleura that could not be diagnosed by conventional bronchoscopy under X-ray fluoroscopy. Even compared with these reported diagnostic yields by CT-guided conventionaldiameter bronchoscopy, our diagnostic yield was high. Therefore, the high diagnostic yield in our study may have been due to the use of an ultrathin bronchoscope in combination with CT. In all 158 lesions examined using this procedure, the sensitivity, specificity, accuracy, positive predictive value, and negative predictive value of the procedure for diagnosing malignancy were $78.1,100,86.7,100$, and $74.7 \%$, respectively.

The evaluation of factors contributing to the diagnosis is important in determining the cases indicated for examination and for improving the diagnostic yield. The lesion distance from the hilum has been suggested as a factor predicting a diagnosis of bronchoscopy (14). In cases of solitary pulmonary nodules, Naidich et al (18) reported a high diagnostic yield in the presence of a bronchus leading to the nodule (CT-bronchus sign). Gaeta et al (19) also reported a higher diagnostic yield in lesions for which the involved bronchus was of the 4th generation or less than in other lesions. Since the ultrathin bronchoscope can be inserted into more peripheral areas for observation, and the pulmonary artery runs along the bronchi in the periphery, Shinagawa et al (20) also noted the CT-artery sign and reported a low diagnostic yield in lesions in the absence of both CT-bronchus and CT-artery signs. Multivariate analysis in their study showed no significant difference, which may have been due to the small number of cases, while analysis in our study revealed that the CT-bronchus and/or artery sign is an important factor contributing to the diagnosis. The observation range by ultrathin bronchoscopy was also a factor contributing to the diagnosis. In addition, since CT was used, the diagnostic yield was $70.7 \%$ even in lesions that cannot be observed by X-ray fluoroscopy. This examination may be particularly useful for lesions (including those not observed by fluoroscopy) in which the involved bronchus or pulmonary artery can be confirmed, and lesions distant from the hilum into which the forceps can be inserted after the confirmation of bronchi of the 6th generation or more.

Pathological evaluation showed a low preparation rate of histological specimens (69.2\%). This was because only blood clots and discharge were obtained, and no tissue was collected, or because the specimens were too small and disintegrated in the process of preparing histological specimens. Therefore, particularly because the diagnostic yield of this procedure for benign diseases is low, an increase in the number of biopsies should also be considered. In this study, 61 lesions were diagnosed by cytology, and 16 of those could not be diagnosed by histological examination and were positive only on cytological diagnosis. Our diagnostic yield was higher than that in the study by Shinagawa et al (20), which may have been due to the combination of 
histopathological examination with cytological examination. Different sample collection methods resulted in varying degrees of accurate diagnosis of lung cancer. An ACCP review (1) showed a BAL-washing diagnostic yield of $43 \%$. In the present study, the BAL-washing diagnostic yield was $29.0 \%$, and there were no cases positive only for BAL. Since the ultrathin bronchoscope itself is wedged in the peripheral bronchus, the infusion of a large amount of physiological saline is difficult. In addition, due to the small channel diameter, the aspiration force itself is weak, and the recovery rate is low. The diagnostic sensitivity of brush cytology was shown to be $54 \%$ by the ACCP review (1), but was $45.9 \%$ in the present study. Due to the small brush used, cell collection may have been inadequate. In this study, compared with these methods, cytological diagnosis of the discharge attached to the forceps was high (68.0\%), and 8 lesions were positive only using this method. The cytological diagnosis of discharge attached to the forceps is a safe method that does not require the collection of new samples. In ultrathin bronchoscopy, when considering the low specimen preparation rate, cytological diagnosis should be performed, including that of the discharge attached to the forceps.

\section{The authors state that they have no Conflict of Interest (COI).}

\section{Acknowledgement}

The authors would like to thank Dr. Jun Kawaguchi (Craft Japan Corporation) for his advice regarding the statistical analyses used in this study.

\section{References}

1. Rivera MP, Mehta AC. Initial diagnosis of lung cancer: ACCP evidence-based clinical practice guidelines (2nd edition). Chest 132: 131S-148S, 2007

2. Wagner U, Walthers EM, Gelmetti W, Klose KJ, von Wichert P. Computer-tomographically guided fiberbronchoscopic transbronchial biopsy of small pulmonary lesions: a feasibility study. Respiration 63: 181-186, 1996.

3. Kurimoto N, Miyazawa T, Okimasa S, et al. Endobronchial ultrasonography using a guide sheath increases the ability to diagnose peripheral pulmonary lesions endoscopically. Chest 126: 959-965, 2004.

4. Schwarz Y, Mehta AC, Ernst A, et al. Electromagnetic navigation during flexible bronchoscopy. Respiration 70: 516-522, 2003.

5. Tanaka M, Kohda E, Satoh M, Yamasawa F, Kawai A. Diagnosis of peripheral lung cancer using a new type of endoscope. Chest 97: 1231-1234, 1990.

6. Tanaka M, Takizawa H, Satoh M, Okada Y, Yamasawa F, Umeda A. Assessment of an ultrathin bronchoscope that allows cytodiagnosis of small airways. Chest 106: 1443-1447, 1994.

7. Hasegawa S, Hitomi S, Murakawa M, Mori K. Development of an ultrathin fiberscope with a built-in channel for bronchoscopy in infants. Chest 110: 1543-1546, 1996.

8. Rooney CP, Wolf K, McLennan G. Ultrathin bronchoscopy as an adjunct to standard bronchoscopy in the diagnosis of peripheral lung lesions. A preliminary report. Respiration 69: 63-68, 2002.

9. Saka H. Ultra-fine bronchoscopy: Biopsy for peripheral lesions. Nippon Rinsho 60 (Suppl 5): 188-190, 2002 (in Japanese).

10. Asano F, Kimura T, Shindou J, Matsuno Y, Mizutani H, Horiba M. Usefulness of CT-guided ultrathin bronchoscopy in the diagnosis of peripheral pulmonary lesions that could not be diagnosed by standard transbronchial biopsy. J Jpn Soc Bronchol 24: 80-85, 2002 (in Japanese).

11. Asano F, Matsuno Y, Matsushita $T$, et al. Transbronchial diagnosis of a pulmonary peripheral small lesion using an ultrathin bronchoscope with virtual bronchoscopic navigation. J Bronchol 9: 108111, 2002.

12. Kobayashi T, Shimamura K, Hanai K, Kaneko M. Computed tomography-guided bronchoscopy with an ultrathin fiberscope. Diagn Ther Endosc 2: 229-232, 1996.

13. Chechani V. Bronchoscopic diagnosis of solitary pulmonary nodules and lung masses in the absence of endobronchial abnormality. Chest 109: 620-625, 1996.

14. Baaklini WA, Reinoso MA, Gorin AB, Sharafkaneh A, Manian P. Diagnostic yield of fiberoptic bronchoscopy in evaluating solitary pulmonary nodules. Chest 117: 1049-1054, 2000.

15. Yamamoto $S$, Ueno $K$, Imamura $F$, et al. Usefulness of ultrathin bronchoscopy in diagnosis of lung cancer. Lung Cancer 46: 43-48, 2004.

16. Tsushima K, Sone S, Hanaoka T, Takayama F, Honda T, Kubo K. Comparison of bronchoscopic diagnosis for peripheral pulmonary nodule under fluoroscopic guidance with CT guidance. Respir Med 100: 737-745, 2006.

17. Heyer CM, Kagel T, Lemburg SP, et al. Transbronchial biopsy guided by low-dose MDCT: a new approach for assessment of solitary pulmonary nodules. AJR Am J Roentgenol 187: 933-939, 2006.

18. Naidich DP, Sussman R, Kutcher WL, Aranda CP, Garay SM, Ettenger NA. Solitary pulmonary nodules. CT-bronchoscopic correlation. Chest 93: 595-598, 1988.

19. Gaeta M, Russi EG, La Spada F, Barone M, Casablanca G, Pandolfo I. Small bronchogenic carcinomas presenting as solitary pulmonary nodules. Bioptic approach guided by CT-positive bronchus sign. Chest 102: 1167-1170, 1992.

20. Shinagawa N, Yamazaki K, Onodera Y, et al. Factors related to diagnostic sensitivity using an ultrathin bronchoscope under CT guidance. Chest 131: 549-553, 2007.

(C) 2011 The Japanese Society of Internal Medicine http://www.naika.or.jp/imindex.html 